\title{
The neutrophil-to-lymphocyte ratio is an effective prognostic marker in localized upper urinary tract urothelial carcinoma treated with nephroureterectomy
}

Mari Ohtaka $^{1 \#}$, Takashi Kawahara ${ }^{1,2 * *}$, Taku Mochizuki ${ }^{1}$, Hiroaki Ishida ${ }^{1}$, Shinnnosuke Kuroda ${ }^{1}$, Moeka Shinbori ${ }^{2}$, Yusuke Hattori ${ }^{1}$, Jun$^{-}$ ichi Teranishi ${ }^{1}$, Kazuhide Makiyama ${ }^{2}$, Yasuhide Miyoshi ${ }^{1}$, Yasushi Yumura ${ }^{1}$, Hiroshi Miyamoto ${ }^{3}$, Masahiro Yao ${ }^{2}$ and Hiroji Uemura $^{1}$

${ }^{1}$ Department of Urology, Yokohama City University Graduate School of Medicine, Japan

${ }^{2}$ Departments of Urology and Renal Transplantation, Yokohama City University Medical Center, Japan

${ }^{3}$ Departments of Pathology and Urology, Johns Hopkins University School of Medicine, USA

\#Equal Contribution

\begin{abstract}
Background: Recent studies have revealed that the neutrophil-to-lymphocyte ratio (NLR) is an independent prognostic factor for several types of malignancies including urological cancers. We herein evaluated the significance of NLR in localized upper urinary tract urothelial carcinoma (UUT-UC) treated with surgery.

Methods: A total of 60 patients who underwent radical nephroureterectomy at our institution from 1995 to 2015 were evaluated. The association between preoperative NLR and the prognosis was analyzed.

Results: The higher NLR $(\geq 2.43)$ group showed a significantly poorer overall survival $(p=0.007)$ than the lower NLR group. There was no association between NLR and recurrence-free survival $(p=0.213)$. High-grade tumors tended to show higher NLRs $(p=0.068)$, which significantly correlated with the cancer grade $(p<0.01)$ and muscle invasion $(\mathrm{p}=0.02)$. In contrast, the NLR did not correlate with lymph node metastasis $(\mathrm{p}=0.69)$. According to a multivariate analysis, the NLR was an independent risk factor for the overall survival $(\mathrm{p}=0.008, \mathrm{HR}=12.194)$.

Conclusions: Preoperative NLR is therefore considered to be a useful biomarker to predict the prognosis of the patients with UUT-UC treated with radical nephroureterectomy.
\end{abstract}

Abbreviations: NLR: Neutrophil to Lymphocyte Ratio, UUT-UC: Upper Urinary Tract Urothelial Carcinoma

\section{Background}

Upper urinary tract urothelial carcinoma (UUT-UC) accounts for approximately $5 \%$ of urothelial cancers [1]. Radical nephroureterectomy is the standard treatment for localized UUT-UC. However, UUT-UC correlates with a high incidence of tumor recurrence and a poor patient outcome. Although the pathological findings, including the tumor size, tumor number, tumor grade, stage, lymph node involvement, and lymphovascular invasion, are thought to be predictors of recurrence [2-5], a new marker that more precisely predicts the prognosis prior to nephroureterectomy is needed to help provide additional treatment, such as neoadjuvant systemic chemotherapy.

Previous studies have shown that inflammation is associated with cancer development and progression. In particular, C-reactive protein as a systemic inflammation marker was reported to be a poor prognostic factor in several types of cancers [6,7]. Others and we have also revealed that neutrophil-to-lymphocyte ratio (NLR) is an independent prognostic factor in various solid malignancies, including renal cell carcinoma, bladder urothelial carcinoma, and prostatic carcinoma [8-13]. Of note, NLR can be easily calculated from complete blood counts (CBCs) in the peripheral blood, and this parameter can be used both pre- and post-operatively. The aim of this study is to evaluate preoperative NLR as a prognostic marker in patients with localized UUT-UC treated with radical nephroureterectomy.

\section{Materials and methods}

\section{Patients}

A total of 60 Japanese patients treated with radical nephroureterectomy for their UUT-UC in Yokohama City University Hospital (Yokohama, Japan) from December 1998 to July 2015 were enrolled in this study. All these patients had a blood test with CBCs preoperatively. Institutional Review Board of Yokohama City University Hospital approved this study. The tumor grade was determined according to the World Health Organization guidelines 1973 and WHO/ISUP (International Society of Urological Pathology) classification. Pathological $T$ stage was adjusted according to the TNM classification. Postoperatively, patients were followed up every 3

Correspondence to: Takashi Kawahara, M.D., Departments of Urology and Renal Transplantation, Yokohama City University Medical Center, 4-57 Urafune-cho, Minami-ku, Yokohama, Kanagawa, 2320024, Japan, Tel: +81-45-261-5656, Fax: +81-45-786-5775, E-mail: takashi_tk2001@yahoo.co.jp

key words: upper urinary tract urothelial carcinoma, neutrophil-to-lymphocyte ratio, NLR, nephroureterectomy

Received: July 05, 2017; Accepted: July 29, 2017; Published: August 02, 2017 
Ohtaka M (2017) The neutrophil-to-lymphocyte ratio is an effective prognostic marker in localized upper urinary tract urothelial carcinoma treated with nephroureterectomy

months for 2 years and thereafter every 6 months by both cystoscopy and computed tomography scans.

NLR was calculated using the neutrophil and lymphocyte counts in CBCs obtained a few days before surgery. We determined the cut-off points of NLR based on the sensitivity and specificity levels derived from area under receiver operator characteristic (AUROC) curves plotted for death or disease progression. None of the patients demonstrated systemic inflammation or blood disease at the time of the blood test. Disease recurrence was defined as local tumor development, including development in the lymph nodes, distant metastasis, and recurrence in the bladder. The data is listed in table 1.

\section{Statistical analysis}

The patients' characteristics and preoperative factors were analyzed by the Mann-Whitney U and chi-square tests, using the Graph Pad Prism software program (Graph Pad Software, La Jolla, CA, USA). The survival duration was defined as the time between the dates of pathological diagnosis and tumor recurrence or death. A log-rank test was performed for comparisons between higher and lower NLR groups. A multivariate analysis was performed using the SPSS software program (IBM, Chicago, IL, USA). P values of $<0.05$ were considered to indicate statistical significance.

\section{Results}

\section{Patients' characteristics}

The patients included 44 males and 16 females. The median age (range) was 70.5 (21-85) years, and the median follow-up duration

Table 1. Baseline patient characteristics

\begin{tabular}{|l|l|}
\hline \multicolumn{1}{|c|}{ Variables } & \multicolumn{1}{|c|}{ number (\%) or (mean, median) } \\
\cline { 2 - 2 } & \\
\hline Number of Pts. & $60(100.0 \%)$ \\
\hline Age (mean, median) & $67.8,70.5$ \\
\hline Gender & \\
\hline Male & $44(73.3 \%)$ \\
\hline Female & $15(35.7 \%)$ \\
\hline Side & \\
\hline Left & $26(43.3 \%)$ \\
\hline Right & $10(16.7 \%)$ \\
\hline Lymphnode metastasis & \\
\hline T stage & \\
\hline is & $5(8.3 \%)$ \\
\hline a & $7(11.7 \%)$ \\
\hline 1 & $16(26.7 \%)$ \\
\hline 2 & $7(11.7 \%)$ \\
\hline 3 & $24(49.0 \%)$ \\
\hline unknown & $1(1.7 \%)$ \\
\hline Low & $14(23.3 \%)$ \\
\hline High & $32(53.3 \%)$ \\
\hline Unknown & $16(26.7 \%)$ \\
\hline Grade1 & $9(15.0 \%)$ \\
\hline Grade2 & $28(46.7 \%)$ \\
\hline Grade3 & $21(35.0 \%)$ \\
\hline Unknown & $2(3.3 \%)$ \\
\hline Recurrence & $31(51.7 \%)$ \\
\hline Bladder & $19(31.7 \%)$ \\
\hline Lung & $7(11.7 \%)$ \\
\hline Liver & $4(6.7 \%)$ \\
\hline Lymphnode & $4(6.7 \%)$ \\
\hline Bone & $2(3.3 \%)$ \\
\hline
\end{tabular}

(range) after surgery was 35 (1-235) months. The characteristics of the patients and the clinicopathologic data, including tumor grade, T stage, and the presence of lymph node metastasis, are summarized in Table 1. During the follow-up period, 31 (51.7\%) patients had recurrence, and the median recurrence-free survival (RFS) was 32 months. Of these patients, 19 (31.7\%) experienced recurrence as a bladder tumor.

\section{NLR value and patient outcomes}

Based on the AUROC curve, the NLR cut-off point was determined to be 2.43 for death (AUROC: 0.690; Figure 1) and 2.35 for recurrence (AUROC: 0.469; figure not shown). A Kaplan-Meier analysis and logrank test revealed that higher NLR was correlated with a significantly lower overall survival (OS) rate compared with lower NLR ( $p=0.007$; Figure 2). However, no significant differences in the NLRs were noted between the non-muscle invasive and muscle invasive groups ( $\mathrm{p}=0.104$ and 0.166 , respectively). The association between NLR and RFS was not statistically significant $(\mathrm{p}=0.213$; Figure 3$)$. Moreover, high NLR was significantly correlated with high tumor grade $(\mathrm{p}=0.07$, low-grade vs high-grade; $p<0.001$, G1 vs G2 vs G3), muscle invasion or not $(\mathrm{p}=0.02)$, and not significant for lymph node metastasis $(\mathrm{p}=0.69)$ (Fig. 4). According to a multivariate analysis, the NLR was an independent risk factor for the overall survival $(\mathrm{p}=0.008, \mathrm{HR}=12.194)$ (Table 2).

\section{Discussion}

Neutrophils play an important role in inducing cytokines to promote tumor progression or invasiveness, and an increasing number of neutrophils results in tumor progression. On the other hand, lymphocytes have a potential anti-tumor activity in the host immune system. Although the detailed mechanisms remain unclear, an increased NLR is thought to be due to the combination of upgrading of tumor progression by neutrophils and downgrading of the host immune system by lymphocytes [14-16].

Prognostic significance of NLR in patients with a variety of solid malignancies have been studied. Some of these studies revealed correlations between NLR and prognosis of patients with UUT-UC who underwent nephroureterectomy [17-20]. In urothelial carcinoma, our previous reports showed the cut-off points as 2.38 for the patients who received radical cystectomy and the other reports in bladder cancer showed similar cut-off point $(2.38-3.0)$ [11]. For UUT-UC, the cut-off points were 2.5 to $2.7[19,20]$. Our results also supported that data. Consistent with these findings, our results demonstrated that high preoperative NLR strongly correlated with worse patient outcomes. However, no correlations between NLR and tumor invasiveness or lymph node metastasis were identified.

Nephroureterectomy is the gold standard for localized UUTUC. However, there is currently no consensus on neoadjuvant or adjuvant systemic chemotherapy. Several studies showed that adjuvant chemotherapy reduced the risk of distant metastasis or intrabladder tumor recurrence. In contrast, there has been no evidence for neoadjuvant chemotherapy. Because of the difficulty in isolating high-risk patients, neoadjuvant chemotherapy may result in overtreatment $[21,22]$. Meanwhile, NLR can be calculated preoperatively, and a high preoperative NLR may therefore suggest the indication of neoadjuvant chemotherapy.

There are several limitations associated with our study. First, the study was retrospective in nature. Second, NLR may be affected by infection, systemic inflammation and medication, while none of the patients in our study had such conditions at the time of blood test before undergoing nephroureterectomy Third, the sample size was relatively small. So, large study is needed to conclude the results. 


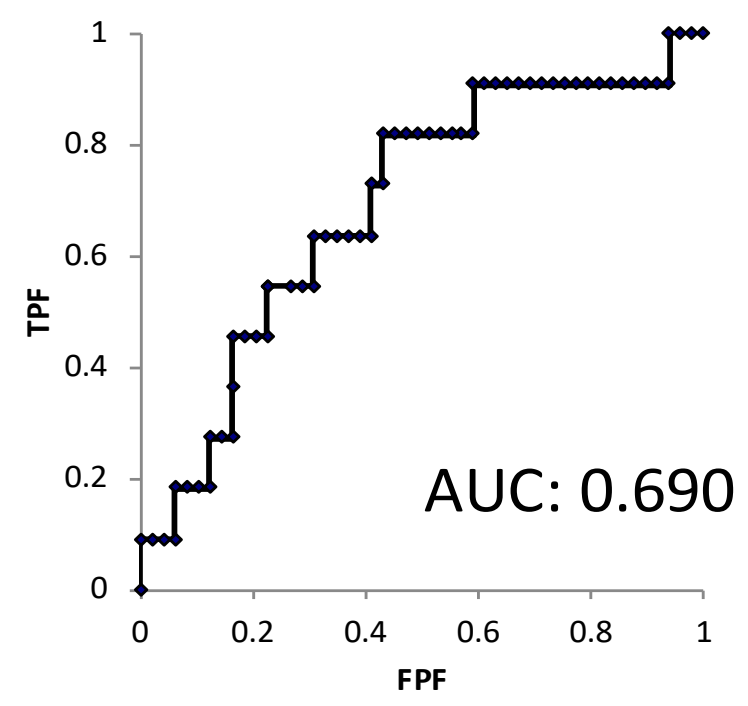

The AUROC of NLR (AUROC:0.690). The NLR cut-off point was determined to be 2.43 for death.

Figure 1. The AUROC of NLR.

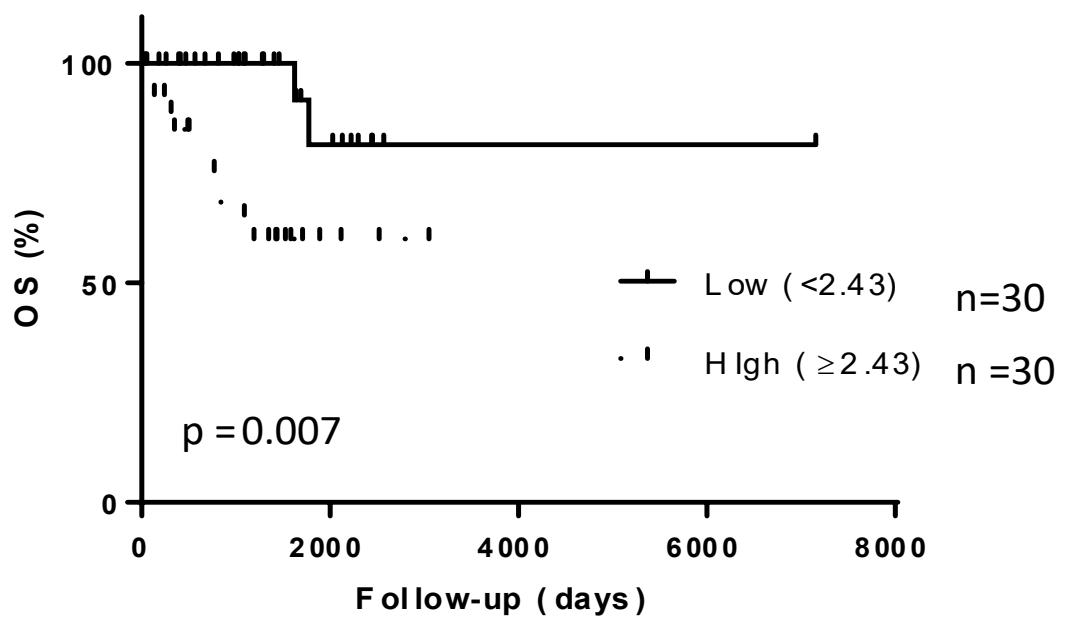

The association of NLR with OS. Higher NLR was correlated with a significantly lower OS rate compared with lower NLR.

Figure 2. The association of NLR with overall survival.

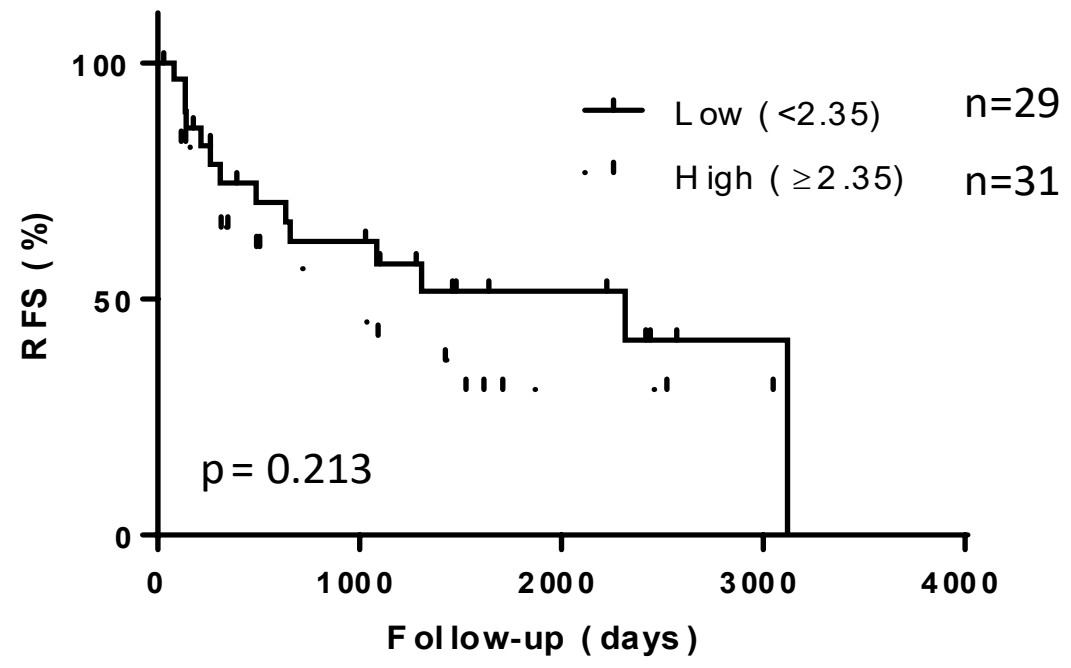

The association of NLR with RFS. The association between NLR and RFS was not statistically significant.

Figure 3. The association of NLR with recurrence-free survival. 
Ohtaka M (2017) The neutrophil-to-lymphocyte ratio is an effective prognostic marker in localized upper urinary tract urothelial carcinoma treated with nephroureterectomy

a

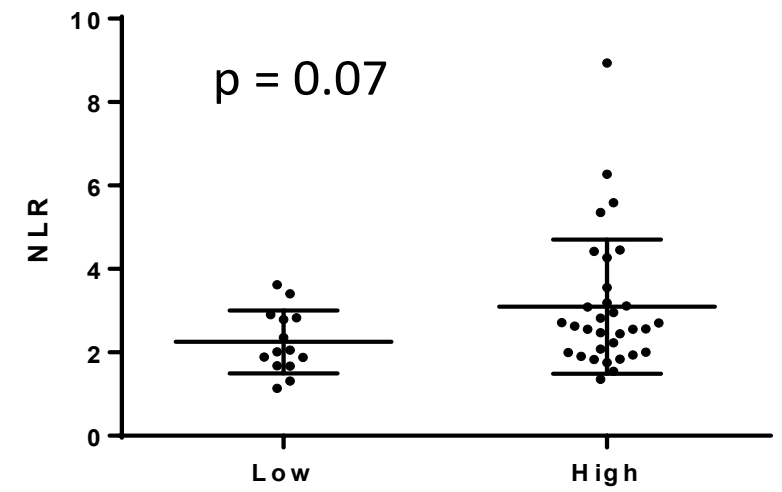

C

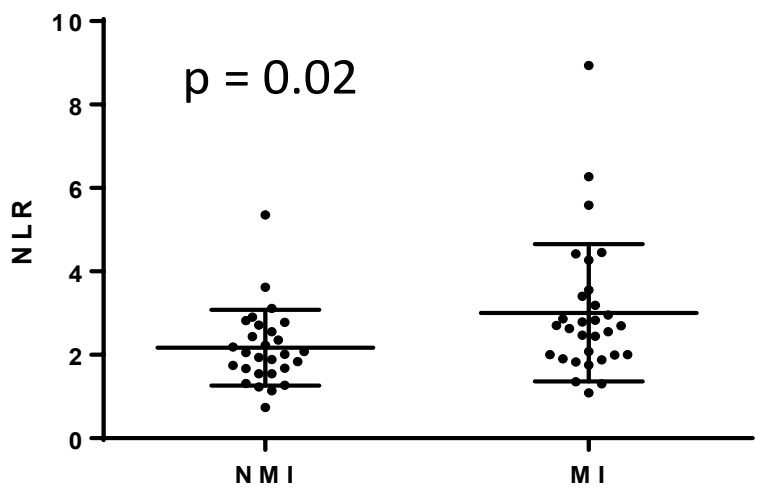

b

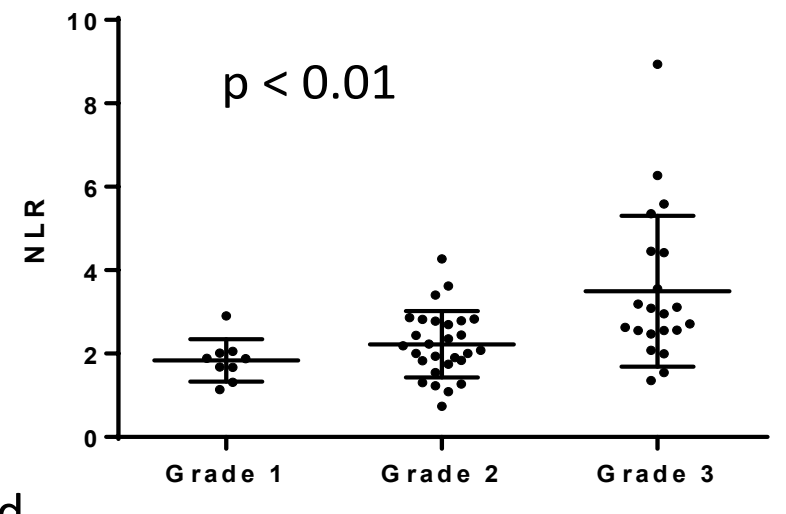

d

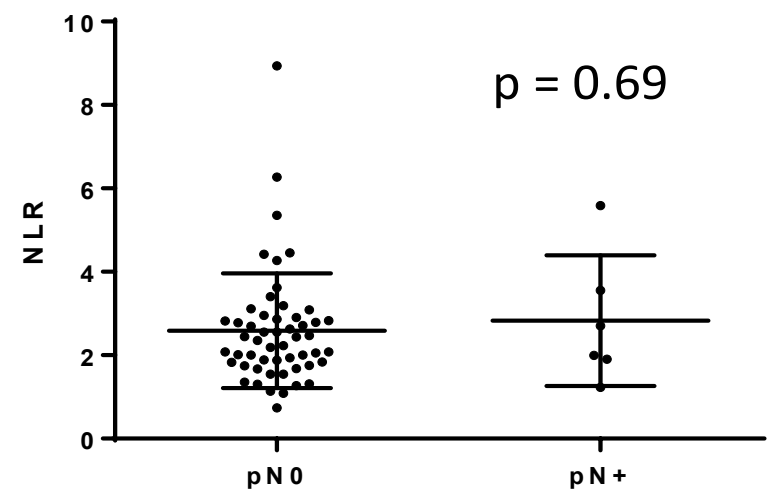

Figure 4. NLR and clinicopathological features, including tumor grade (a, b), muscle invasiveness (c), and lymph node metastasis (d).

Table 2. Multivariate analysis

\begin{tabular}{|c|c|c|c|}
\hline & P value & HR & $\mathbf{9 8 \% C I}$ \\
\hline Gender (male) & 0.371 & 0.687 & $0.121-3.894$ \\
\hline Age $(>70)$ & 0.050 & 0.190 & $0.036-1.000$ \\
\hline NLR $(\geq 2.43)$ & 0.008 & 12.194 & $1.930-77.056$ \\
\hline Muscle invasive & 0.404 & 1.903 & $0.420-8.630$ \\
\hline $\mathrm{pN}+$ & 0.029 & 6.398 & $1.208-33.894$ \\
\hline
\end{tabular}

In conclusion, NLR is a potential biomarker to predict the survival in UUT-UC patients who have undergone radical nephroureterectomy. Patients with high preoperative NLR are suggested to be candidates for additional chemotherapy pre- and/or post-operatively.

\section{Authors' contributions}

Conceived and designed the experiments: MO, TK, HI, SK, MS, KM ,YY, HM, HU. Performed the experiments: TM, HI, YH, JT, YM. Wrote the paper: MO, TK, HM, MY, HU. All authors have read and approved the manuscript

\section{Availability of supporting data}

Due to ethical restrictions, the raw data underlying this paper is available upon request to the corresponding author.

\section{Funding declaration}

Grants from KAKENHI grants (16K20152) from the Ministry of Education, Culture, Sports, Science and Technology of Japan.
Institutional Review Board of Yokohama City University Hospital approved this study.

\section{References}

1. Siegel R, Ma J, Zou Z, Jemal A (2014) Cancer statistics, 2014. CA Cancer J Clin 64 9-29. [Crossref]

2. Margulis V, Shariat SF, Matin SF, Kamat AM, Zigeuner R, et al. (2009) Upper Tract Urothelial Carcinoma CollaborationThe Upper Tract Urothelial Carcinoma C. Outcomes of radical nephroureterectomy: a series from the Upper Tract Urothelial Carcinoma Collaboration. Cancer 115: 1224-1233. [Crossref]

3. Rink M, Sjoberg D, Comploj E, Margulis V, Xylinas E, et al. (2012) Risk of cancerspecific mortality following recurrence after radical nephroureterectomy. Ann Surg Oncol 19: 4337-4344. [Crossref]

4. Azemar MD, Comperat E, Richard F, Cussenot O, Roupret M (2011) Bladder recurrence after surgery for upper urinary tract urothelial cell carcinoma: frequency, risk factors, and surveillance. Urol Oncol 29: 130-136. [Crossref]

5. Kimura S, Miki J, Tsuduki S, Aikawa K, Nishikawa H, et al. (2015) [Risk Factors for Intravesical Recurrence after Surgical Management of Urotherial Carcinoma of the Upper Urinary Tract]. Nihon Hinyokika Gakkai Zasshi 106: 231-237. [Crossref]

6. Ito H, Shioi K, Murakami T, Takizawa A, Sano F, et al. (2012) C-reactive protein in patients with advanced metastatic renal cell carcinoma: usefulness in identifying patients most likely to benefit from initial nephrectomy. BMC Cancer 12: 337. [Crossref]

7. Ishiguro H, Kawahara $\mathrm{T}$ (2014) Nonsteroidal anti-inflammatory drugs and prostatic diseases. Biomed Res Int 2014: 436123. [Crossref]

8. Pichler M, Hutterer GC, Stoeckigt C, Chromecki TF, Stojakovic T, et al. (2013) Validation of the pre-treatment neutrophil-lymphocyte ratio as a prognostic factor in a large European cohort of renal cell carcinoma patients. Br J Cancer 108: 901-907. [Crossref] 
Ohtaka M (2017) The neutrophil-to-lymphocyte ratio is an effective prognostic marker in localized upper urinary tract urothelial carcinoma treated with nephroureterectomy

9. Cho H, Hur HW, Kim SW, Kim SH, Kim JH, et al. (2009) Pre-treatment neutrophil to lymphocyte ratio is elevated in epithelial ovarian cancer and predicts survival after treatment. Cancer Immunol Immunother 58: 15-23. [Crossref]

10. Perez DR, Baser RE, Cavnar MJ, Balachandran VP, Antonescu CR, et al. (2013) Blood neutrophil-to-lymphocyte ratio is prognostic in gastrointestinal stromal tumor. Ann Surg Oncol 20: 593-599. [Crossref]

11. Kawahara T, Furuya K, Nakamura M, Sakamaki K, Osaka K, et al. (2016) Neutrophilto-lymphocyte ratio is a prognostic marker in bladder cancer patients after radical cystectomy. BMC Cancer 16: 185. [Crossref]

12. Kawahara T, Yokomizo Y, Ito Y, Ito H, Ishiguro H, et al. (2016) Pretreatment neutrophilto-lymphocyte ratio predicts the prognosis in patients with metastatic prostate cancer. BMC Cancer 16: 111. [Crossref]

13. Kawahara T, Fukui S, Sakamaki K, Ito Y, Ito H, et al. (2015) Neutrophil-to-lymphocyte ratio predicts prostatic carcinoma in men undergoing needle biopsy. Oncotarget 6 : 32169-32176. [Crossref]

14. Terzić J, Grivennikov S, Karin E, Karin M (2010) Inflammation and colon cancer. Gastroenterology 138: 2101-2114. [Crossref]

15. Lin EY, Pollard JW (2004) Role of infiltrated leucocytes in tumour growth and spread. Br J Cancer 90: 2053-2058. [Crossref]

16. An X, Ding PR, Li YH, Wang FH, Shi YX, et al. (2010) Elevated neutrophil to lymphocyte ratio predicts survival in advanced pancreatic cancer. Biomarkers 15: 516522. [Crossref]
17. Sung HH, Jeon HG, Jeong BC, Seo SI, Jeon SS, et al. (2015) Clinical significance of prognosis using the neutrophil-lymphocyte ratio and erythrocyte sedimentation rate in patients undergoing radical nephroureterectomy for upper urinary tract urothelial carcinoma. BJU Int 115: 587-594. [Crossref]

18. Luo HL, Chen YT, Chuang YC, Cheng YT, Lee WC, et al. (2014) Subclassification of upper urinary tract urothelial carcinoma by the neutrophil-to-lymphocyte ratio (NLR) improves prediction of oncological outcome. BJU Int 113(5b): E144-149. [Crossref]

19. Dalpiaz O, Ehrlich GC, Mannweiler S, Hernandez JM, Gerger A, et al. (2014) Validation of pretreatment neutrophil-lymphocyte ratio as a prognostic factor in a European cohort of patients with upper tract urothelial carcinoma. BJU Int 114: 334-339. [Crossref]

20. Azuma T, Matayoshi Y, Odani K, Sato Y, Sato Y, et al. (2013) Preoperative neutrophillymphocyte ratio as an independent prognostic marker for patients with upper urinary tract urothelial carcinoma. Clin Genitourin Cancer 11: 337-341. [Crossref]

21. Bamias A, Deliveliotis C, Fountzilas G, Gika D, Anagnostopoulos A, et al. (2004) Adjuvant chemotherapy with paclitaxel and carboplatin in patients with advanced carcinoma of the upper urinary tract: a study by the Hellenic Cooperative Oncology Group. J Clin Oncol 22: 2150-2154. [Crossref]

22. Soga N, Arima K, Sugimura Y (2008) Adjuvant methotrexate, vinblastine, adriamycin, and cisplatin chemotherapy has potential to prevent recurrence of bladder tumors after surgical removal of upper urinary tract transitional cell carcinoma. Int J Urol 15: 800803. [Crossref]

Copyright: ( 2017 Ohtaka M. This is an open-access article distributed under the terms of the Creative Commons Attribution License, which permits unrestricted use, distribution, and reproduction in any medium, provided the original author and source are credited. 